\title{
Impact of Information Sharing Regarding Customer Returns Ratio on Optimal Sales Strategy under E-commerce
}

\author{
Yuta Saito, Etsuko Kusukawa* \\ Graduate School of Engineering, Osaka Prefecture University, Osaka, Japan
}

(Received: January 2, 2015 / Revised: March 30, 2015 / Accepted: April 6, 2015)

\begin{abstract}
A correct information of customer returns ratio under e-commerce is not always shared between supply chain (SC) members. Also, it is important issue for SC members to handle the unsold products in a market. This paper discusses the impact of information sharing of customer returns ratio on an optimal sales strategy including resale of customer returns and buyback policy for a SC under e-commerce with a manufacturer and a retailer. A retailer sells a single product and resells the resalable customer returns in the same market. A manufacturer produces the products and buys back the unsold products as to their quality from the retailer. The integrated SC (ISC) determines the optimal product order quantity to maximize the expected profit of the whole SC. The decentralized SC (DSC) makes the optimal decisions for order quantity and the wholesale price of products to maximize the expected profit of each SC member. The effect of information sharing is discussed between SC members under ISC and DSC. The analysis numerically investigates how information sharing of the returns ratio affects the optimal decision and the expected profits under ISC and DSC. Besides, effect of SC coordination to encourage the shift to ISC is discussed.
\end{abstract}

Keywords: E-Commerce, Information Sharing of Customer Returns Ratio, Quality of Returns, Returns Handling, Supply Chain Coordination

* Corresponding Author, E-mail: kusukawa@eis.osakafu-u.ac.jp

\section{INTRODUCTION}

For recent years, a share in product sales under an e-commerce environment has been increasing worldwide. However, online sales tend to have a higher returns ratio than brick-and-mortar sales (Lee, 2001, Vlachos and Dekker, 2003; Choi an Yan, 2004; Lee, 2007, Kusukawa et al., 2009; Chen and Bell, 2009; Chen, 2011). Also, correct information regarding customer returns ratio is not always shared between supply chain (SC) members (Chen, 2011). Moreover, it is important issue for $\mathrm{SC}$ members to handle the unsold products and customers returns in a market.

In a supply chain management, it is necessary to determine the optimal operations to establish a $\mathrm{SC}$ to obtain its profitability. In a decentralized SC, all members in the SC determine the optimal operations so as to maximize their profits. As one of the optimal decisionmaking approaches under a decentralized SC, the Stackelberg game has been adopted in several previous papers. In the Stackelberg game, there is a single leader of the decision-making and a single (multiple) follower(s) of the decision-making of the leader. The leader of the decision-making determines the optimal strategy so as to maximize the leader's (expected) profit. The follower(s) of the decision-making determine(s) the optimal strategy so as to maximize the follower(s)'s (expected) profit under the optimal strategy determined by the leader of 
the decision-making (Aust and Buscher, 2012; Berr, 2011; Cachon and Netessine, 2004; Cai et al., 2009; Hu et al.).

Also, in a supply chain management, the optimal decisions under an integrated supply chain maximizing the whole supply chain's expected profit can bring the more expected profit to the whole supply chain than those under a decentralized supply chain maximizing the expected profit of each member in a supply chain. So, from the aspect of the total optimization in supply chain management, it is preferable for all members in supply chain to shift the optimal decisions under the integrated supply chain. In this case, it is the absolute requirement for all members under the integrated supply chain to obtain the more expected profits than those under the decentralized supply chain. In order to achieve the increases in profits of all members under the integrated supply chain, a variety of supply chain coordination approaches between all members have been discussed by Cachon and Netessine (2004), Chauhan and Proth (2005), Nagarajan and Sosic (2008), Du et al. (2011).

This paper focuses on an optimal inventory strategy in a supply chain in order to provide guiding principles to handle the unsold products and the customer returns after the end of the selling period of the normal sales market. Some previous papers have dealt with the unsold products including customer returns in the normal sales market.

Choi and Yan (2004 presented an optimal sales strategy for a supply chain with normal sales market, secondary market and salvage market. A production quantity of a single product and the unit clearance sales price of the unsold products in the normal sales market were optimized under both decentralized supply chain (DSC) and integrated supply chain (ISC) by theoretical analysis. However, any supply chain coordination between supply chain members was not discussed.

Vlachos and Dekker (2003) proposed the optimal sales strategy for product order quantity in a supply chain under an e-commerce, considering several options to handle customer returns from a normal sales market.

Kusukawa, Niimi and Arizono (2009) presented optimal decision approaches for product order quantity under both DSC and ISC with normal sales market, secondary market and salvage market. They also verified that the optimal decision under ISC brought more expected profit to the entire supply chain. However, the supply chain members consisting of a retailer and a manufacturer could not be guaranteed more profits under ISC. When the optimal decisions under ISC are adopted, it is the absolute requirement for all members under ISC to obtain more expected profits than those under DSC. Also, the profitability of clearance sales was not discussed.

Lee (2001) presented a clearance sales strategy for a distribution channel consisting of a supplier, a retailer and a discount sales outlet (DSO). An optimal sales strategy for product order quantity, markdown price ratio and a return quantity in a supply chain with a normal sales market and a markdown sales market were verified by theoretical analysis. Also, a retailer-DSO coordination was analyzed by changing the supplier's unit wholesale price, the retailer's unit selling price, the unit return value and the unit leftover whole sale price. However, profit sharing approach between supply chain members to guarantee more profit for the optimal decisions under ISC was not discussed here.

Lee (2007) presented an optimal sales strategy for product order quantity and a markdown price ratio in a supply chain with a normal sales market and a markdown sales market. Supply chain members consist of a manufacturer and a discount sales outlet (DSO). The discount outlet determines a markdown sales quantity and a markdown sales ratio. Also, a manufacturer-DSO coordination was analyzed by changing the manufacturer's constant demand ratio, the manufacturer's unit maximum selling price. However, not only the profitability of buyback for the unsold products after the selling period of markdown market, but also any profit sharing approach between supply chain members to guarantee more profit for the optimal decisions under ISC were not discussed.

Chen and Bell (2009) addressed the simultaneous determination of price and inventory replenishment when customers returned product to the firm. This previous study considered that the quantity of returned product affected both the product quantity sold in a market and the retail price. Results were shown for optimal prices and order quantities in single and multi-period problems, with and without uncertainty in demand.

Chen (2011) investigated how sharing of customers return information affected the expected profits of SC members and the optimal sales strategies under DSC and ISC. Also, this previous study discussed the optimal sales strategy policy with buyback policy. However, unsold inventories and returned products are bought back at the same buyback price. In terms of quality of them, it is necessary to distinguish these two products.

Focusing on E-business based on the previous papers mentioned above, this paper discusses the following situation: suppose that firms market a new product that has a limited product lifetime in a SC under e-commerce. Under the situation, they may face not only the unsold products in the normal sales market, but also customer returns in the market. In order to solve those considerable topics on E-business, this paper incorporates the following topics into a SC under e-commerce: (i) resale of customer returns without/with information sharing of customer returns ratio between SC members, (ii) buyback policy without/with difference of quality between the unsold products in a market and the customer returns in the market and (iii) supply chain coordination (SCC) based on game theory in order to encourage the shift to the decision-making under ISC from that under DSC.

This paper tries to provide the following contributions for both academic researchers and real-world policymakers regarding inventory management of a SC under e-commerce: 
- Presentation of theoretical analysis to verify how the combination of topics (i)-(iii) not only affects the profitability to SC members and the whole SC, but also can bring more profitability to all of them;

- Presentation of SCC approach to guarantee the more expected profits of SC members under ISC's optimal sales strategy than under DSC's one.

Concretely, this paper discusses a SC under e-commerce consisting of a manufacturer and a retailer and verifies how information sharing regarding customer returns ratio affect an optimal sales strategy including resale of product returns as to a manufacturer's buyback policy in a SC under e-commerce. A retailer not only sells a single of products, but also resells the resalable products returned from customers in the same market. A manufacturer produces the products and buys back the unsold products without and with considering the difference of their quality from the retailer. Two types of decision-making in SCs are proposed. (1) ISC where the optimal decision for product order quantity is made to maximize the expected profit of the whole SC. (2) DSC where the optimal decisions for product order quantity and the product wholesale price are made to maximize the expected profit of each SC member. The retailer handles customer returns directly, so can recognize the correct information of customer returns ratio. In ISC, the correct information of returns ratio is always shared between SC members. In DSC, a manufacturer can get the correct returns ratio only if the retailer shares it with the manufacturer. Otherwise, the manufacturer estimates the customer returns ratio from the past data.

The analysis numerically investigates how information sharing of the customer returns ratio affects the optimal sales strategies and the expected profits under ISC and DSC as to the state of buyback policy. Also, the results under ISC are compared with those under DSC. Here, the profitability of information sharing of the customer returns ratio is evaluated by comparing the expected profits under each SC without information sharing with those with it. Moreover, SCC to guarantee the profit improvement for each SC member under ISC is discussed. Concretely, the wholesale price and the buyback prices of the unsold products and the un-reusable products are coordinated between SC members as Nash bargaining solutions. The profitability of SCC on SC members under ISC is verified by comparing with their expected profits under DSC.

The purpose of this paper is to enhance understanding and provide the following managerial insights from theoretical research outcomes: the combination of (i) resale of customer returns with information sharing of customer returns ratio between SC members, (ii) buyback policy with difference of quality between the unsold products and the customer returns in the market and (iii) supply chain coordination between SC members in ISC under e-commerce enables to guarantee not only the achievement of the total optimization to maxi- mize the whole SC, but also the more profits of $\mathrm{SC}$ members under ISC than under DSC. Thus, it is expected that research outcomes in this paper enable to link strongly to E-business and those beneficial management. For this reason, this paper can contribute not only informative motivation, but also one optimal solution to construct and manage beneficially a supply chain under e-com-merce for researchers and policymakers regarding the optimal strategy in supply chains.

The rest of this paper is organized as follows: in Section 2, notation used in mathematical model in this paper is defined. Section 3 presents model descriptions of a SC under e-commerce including the operational flows and the model assumptions. Section 4 formulates the expected profits in the SC under e-commerce, considering not only impact of information sharing regarding customer returns ratio, but also difference of quality of returned products. Section 5 presents the optimal sales strategy under ISC, considering difference of quality of returned products. Section 6 presents the optimal sales strategy under DSC, considering not only impact of information sharing regarding customer returns ratio, but also difference of quality of returned products. Section 7 shows the results of numerical examples for the optimal sales strategies under ISC and DSC. Also, section 7 discusses the profitability of supply chain coordination to ISC under e-commerce, as to case without/with information sharing regarding customer returns ratio. Moreover, Section 7 illustrates the numerical outcomes of this paper. In Section 8, conclusions, managerial insights and future researches for this paper are summarized.

\section{NOTATIONS}

$Q:$ order quantity of product, referred to order quantity $w$ : wholesale price per product

$p:$ retail price per product

$g$ : shortage penalty cost per unsatisfied demand of product

$c$ : production cost per product

$b$ : buyback price per unsold product

$b_{r}$ : buyback price per returned and un-resold product

$c_{r}$ : retailer's handling cost per returned product

$c_{m}$ : manufacturer's handling cost per products bought back from a retailer

$s_{r}$ : salvage value per product bought back by a manufacturer

$x$ : product demand in a market

$f(x)$ : probability density function of product demand

$F(x)$ : distribution function of product demand

$\alpha^{t}$ : correct returns ratio of products from a market $\left(0 \leq \alpha^{t} \leq 1\right)$

$\alpha^{e}$ : returns ratio of products estimated by a manufacturer when a retailer and a manufacturer make no information sharing of return ratio of products, referred to the estimated returns ratio $\left(0 \leq \alpha^{e} \leq 1\right)$ 
$g(\alpha)$ : probability density function of returns ratio $\alpha$ of product

$k$ : resalable ratio of returned product in a market $(0 \leq k \leq 1)$

$i$ : index to express the state of buyback policy; $i=1$ indicates buyback policy without difference of quality (buyback policy 1), $i=2$ indicates buyback policy with difference of quality (buyback policy 2)

$j$ : index to express the state of returns ratio of product; $j=t$ indicates the correct returns ratio, $j=e$ indicates the estimated returns ratio of product

$Q_{C}$ : optimal order quantity of product under ISC

$Q_{D}^{i}\left(\alpha^{j}\right)(i=1,2, j=t, e)$ : optimal order quantity under DSC in states of buyback policy $i(=1,2)$ and returns ratio $\alpha^{j}(j=t, e)$

$w_{D}^{j}\left(\alpha^{j}\right)(i=1,2, j=t, e)$ : wholesale price under DSC in states of buyback policy $i(=1,2)$ and returns ratio $\alpha^{j}(j=t, e)$

$w^{N}\left(\alpha^{j}\right)(j=t, e)$ : wholesale price of product with SCC in state of returns ratio $\alpha^{j}(j=t, e)$

$b^{N}\left(\alpha^{j}\right)(j=t, e)$ : buyback price per unsold product with SCC in state of returns ratio $\alpha^{j}(j=t, e)$

$b_{r}^{N}\left(\alpha^{j}\right)(j=t, e)$ : buyback price per returned and unresold product with SCC in state of returns ratio $\alpha^{j}(j=t, e)$

$E\left[\pi_{R}^{i}\right]$ : the expected profit of a retailer in buyback policy $i(=1,2)$

$E\left[\pi_{M}^{i}\right]$ : the expected profit of a manufacturer in buyback policy $i(=1,2)$

$E\left[\pi_{S}\right]:$ the expected profit of the whole SC

$V_{M}^{i}(i=1,2)$ : the value of information sharing of returns ratio for a manufacturer

$V_{R}^{i}(i=1,2)$ : the value of information sharing of returns ratio for a retailer

\section{MODEL DESCRIPTIONS}

\subsection{Operational Flows of SC}

This paper considers a SC consisting of a retailer and a manufacturer. The operational flows of SC is shown as follows: A retailer orders a single of products to a manufacturer, considering the uncertainties in demand of products in a market. A manufacturer produces the products and sells the products to a retailer. The retailer sells the products in a market during a single period. When the order quantity of products does not satisfy the demand of products, the retailer incurs the shortage penalty cost for the unsatisfied product. While the products are sold in the market within the selling period, the products are returned to the retailer from the market at a specific return rate. The retailer inspects returned products. After the restoration, the reusable returned products can be resold in a certain ratio in the same market. In this case, the retailer incurs the handling cost per returned product. The manufacturer buys back both the unsold products and the un-resold returned products without and with considering difference of quality from the retailer. In this case, the unit buy back price of the unsold products is distinguished from that of the unresold retuned products due to the difference of quality. The manufacturer sells the both unsold products and the un-resold retuned products in a disposal market.

\subsection{Model Assumptions}

(i) A single of products such as mobile phone and personal computer is considered. Product demand $x$ in a market follows a probability distribution with the probability density function $f(x)$ and the distribution function $F(x)$.

(ii) It is allowed that the return for the same product is acceptable once only to the retailer.

(iii) It is assumed that under ISC that both a retailer and a manufacturer know the correct returns ratio of products. Meanwhile, it is assumed under DSC that only a retailer knows the correct returns ratio of products since the retailer accepts the returned products directly from the market. The manufacturer can obtain the correct information of returns ratio of products when a retailer and a manufacturer make information sharing of return ratio of products. Otherwise, the manufacturer estimates the returns ratio of products based on the lowest limit of returns ratio $\alpha_{L}$, the upper limit of returns ratio $\alpha_{U}$ and the probability density function of the returns ratio of products $g(\alpha)$ which the manufacture have obtained from the past data through the transaction with the retailer. In this case, the returns ratio is estimated as

$$
\alpha^{e}=\int_{\alpha_{L}}^{\alpha_{U}} \alpha g(\alpha) d \alpha .
$$

(iv) The following conditions:

$$
\begin{aligned}
& p>w>c \geq g \geq s_{r}>c_{r} \geq c_{m}, w>b>b_{r}, \\
& 0 \leq \alpha^{j} \leq 1(j=t, e), 0 \leq k \leq 1
\end{aligned}
$$

are satisfied so as to guarantee profitable operations regarding a retailer, a manufacturer and the whole $\mathrm{SC}$ under e-commerce.

\section{EXPECTED PROFITS in SC, CONSID- ERING IMPACT OF INFORMATION SHARING REGARDING CUSTOMER RETURNS RATIO}

\subsection{Expected Profits of a Retailer and a Manufac- turer in Case of Buy Back Policy without Dif- ference of Quality (Buyback Policy 1)}

From 3.1 and 3.2, the profit of a retailer consists of 
both the sale and the resale of product in a market, the buyback income from a manufacturer, the procurement cost of product, the handling cost of the returned products and the shortage penalty cost for unsatisfied demand of product. Taking the expected value of demand $x$, the expected profit of the retailer $E\left[\pi_{R, 1}\left(w, Q(w) \mid \alpha^{j}\right)\right]$ for wholesale price $w$, and order quantity $Q(w)$ under returns ratio $\alpha^{j}(j=t, e)$ is formulated as

$$
\begin{aligned}
E & \left.\pi_{R}^{1}\left(w, Q(w) \mid \alpha^{j}\right)\right](j=t, e) \\
& =p\left(1-\alpha^{j}\right) \int_{0}^{Q(w)} x f(x) d x+p\left(1-\alpha^{j}\right) Q(w) \int_{Q(w)}^{\infty} f(x) d x \\
& +\left(p k-c_{r}\right) \alpha^{j} \int_{0}^{Q(w)} x f(x) d x+\left(p k-c_{r}\right) \alpha^{j} Q(w) \int_{Q(w)}^{\infty} f(x) d x \\
& +b \int_{0}^{Q(w)}(Q(w)-x) f(x) d x \\
& +b(1-k) \alpha^{j} \int_{0}^{Q(w)} x f(x) d x+b(1-k) \alpha^{j} Q(w) \int_{Q(w)}^{\infty} f(x) d x \\
& -w Q(w)-g \int_{Q(w)}^{\infty}(x-Q(w)) .
\end{aligned}
$$

Next, the profit of a manufacturer consists of the product wholesales, product sales in a disposal market, the buyback cost to a retailer, and the handling costs of the products bought back from a retailer the production cost of product. Taking the expected value of demand $x$, the expected profit of the manufacturer $E\left[\pi_{M}(w, Q(w))\right.$ $\left.\alpha^{j}\right)$ ] for wholesale price $w$, buyback price $b$ and order quantity $Q(w)$ under returns ratio $\alpha^{j}(j=t, e)$ is formulated as

$$
\begin{aligned}
E & \left.\pi_{M}^{1}\left(w, Q(w) \mid \alpha^{j}\right)\right](j=t, e) \\
& =(w-c) Q(w) \\
& +\left(s_{r}-b-c_{m}\right) \int_{0}^{Q(w)}(Q(w)-x) f(x) d x \\
& +\left(s_{r}-b-c_{m}\right)(1-k) \alpha^{j} \int_{0}^{Q(w)} x f(x) d x \\
& +\left(s_{r}-b-c_{m}\right)(1-k) \alpha^{j} Q(w) \int_{Q(w)}^{\infty} f(x) d x .
\end{aligned}
$$

\subsection{Expected Profits of a Retailer and a Manufac- turer in Case of Buy Back Policy with Differ- ence of Quality (Buyback Policy 2)}

From 3.1 and 3.2, the profit of a retailer consists of both the sale and the resale of product in a market, the buyback income considering the difference of quality from a manufacturer, the procurement cost of product, the handling cost of the returned products and the shortage penalty cost for unsatisfied demand of product. Concretely, taking the expected value of demand $x$, the expected the expected profit of the retailer $E\left[\pi_{R}^{2}(w, Q(w) \mid\right.$ $\left.\left.\alpha^{j}\right)\right](j=t, e)$ for wholesale price $w$, and order quantity $Q(w)$ under returns ratio $\alpha^{j}$ is formulated as

$$
E\left[\pi_{R}^{2}\left(w, Q(w) \mid \alpha^{j}\right)\right](j=t, e)
$$

$$
\begin{aligned}
& =p\left(1-\alpha^{j}\right) \int_{0}^{Q(w)} x f(x) d x+p\left(1-\alpha^{j}\right) Q(w) \int_{Q(w)}^{\infty} f(x) d x \\
& +\left(p k-c_{r}\right) \alpha^{j} \int_{0}^{Q(w)} x f(x) d x+\left(p k-c_{r}\right) \alpha^{j} Q(w) \int_{Q(w)}^{\infty} f(x) d x \\
& +b \int_{0}^{Q(w)}(Q(w)-x) f(x) d x+b_{r}(1-k) \alpha^{j} \int_{0}^{Q(w)} x f(x) d x \\
& -w Q(w)-g \int_{Q(w)}^{\infty}(x-Q(w)) .
\end{aligned}
$$

Next, the profit of a manufacturer consists of the product wholesales, product sales in a disposal market, the buyback cost considering the difference of quality to a retailer, the handling costs of the products bought back from a retailer and the production cost of product. Taking the expected value of demand $x$, the expected profit of the manufacturer $E\left[\pi_{M}^{2}\left(w, Q(w) \mid \alpha^{j}\right)\right](j=t, e)$ for wholesale price $w$ and order quantity $Q(w)$ under returns ratio $\alpha^{j}$ is formulated as

$$
\begin{aligned}
E & {\left[\pi_{M}^{2}\left(w, Q(w) \mid \alpha^{j}\right)\right] } \\
& =(w-c) Q(w)+\left(s_{r}-c_{m}\right) \int_{0}^{Q(w)}(Q(w)-x) f(x) d x \\
& +\left(s_{r}-c_{m}\right)(1-k) \alpha^{j} \int_{0}^{Q(w)} x f(x) d x \\
& +\left(s_{r}-c_{m}\right)(1-k) \alpha^{j} Q(w) \int_{Q(w)}^{\infty} f(x) d x \\
& -b \int_{0}^{Q(w)}(Q(w)-x) f(x) d x \\
& -b_{r}(1-k) \alpha^{j} \int_{0}^{Q(w)} x f(x) d x \\
& -b_{r}(1-k) \alpha^{j} Q(w) \int_{Q(w)}^{\infty} f(x) d x .
\end{aligned}
$$

\subsection{Expected Profit of the Whole SC}

The expected profit of the whole SC under returns ratio $\alpha^{j}(j=t, e)$ is calculated from the sum of the expected profits of the retailer and the manufacturer in Buyback Policy $i(=1,2)$ under $\alpha^{j}$, using Eqs. (2) and (3) in Buyback Policy 1 and Eqs. (4) and (5) in Buyback Policy 2 as follows:

$$
\begin{aligned}
& E\left[\pi_{S}\left(Q \mid \alpha^{j}\right)\right](j=t, e) \\
& =E\left[\pi_{R}^{i}\left(w, Q(w) \mid \alpha^{j}\right)\right]+E\left[\pi_{M}^{i}\left(w, Q(w) \mid \alpha^{j}\right)\right](i=1,2 ; j=t, e) \\
& =E\left[\pi_{R}^{i}\left(Q \mid \alpha^{j}\right)\right]+E\left[\pi_{M}^{i}\left(Q \mid \alpha^{j}\right)\right](i=1,2 ; j=t, e) \\
& =p\left(1-\alpha^{j}\right) \int_{0}^{Q} x f(x) d x+p\left(1-\alpha^{j}\right) Q \int_{Q}^{\infty} f(x) d x \\
& +\left(p k-c_{r}\right) \alpha^{j} \int_{0}^{Q} x f(x) d x+\left(p k-c_{r}\right) \alpha^{j} Q \int_{Q}^{\infty} f(x) d x \\
& +\left(s_{r}-c_{m}\right) \int_{0}^{Q}(Q-x) f(x) d x \\
& +\left(s_{r}-c_{m}\right)(1-k) \alpha^{j} \int_{0}^{Q} x f(x) d x \\
& +\left(s_{r}-c_{m}\right)(1-k) \alpha^{j} Q \int_{Q}^{\infty} f(x) d x \\
& -c Q-g \int_{Q}^{\infty}(x-Q) .
\end{aligned}
$$


From Eq. (6), it can be seen that the terms of both wholesales of product and buyback of the unsold products and the returned and un-resold are canceled out between the manufacturer and the retailer under Buyback Policy $i(=1,2)$ when the expected profit of the whole SC is calculated. Therefore, the expected profit of the whole SC under returns ratio $\alpha^{j}(j=t, e)$ is not affected by Buyback Policy $i(=1,2)$. This indicates that the expected profit of the whole SC under returns ratio $\alpha^{j}(j=t, e)$ calculated in Buyback Policy 1 is same as that in Buyback Policy 2. Also, Eq. (6) shows that the expected profit of the whole SC is a function in terms of order quantity $Q$.

\section{OPTIMAL SALES STRATEGY UNDER ISC}

From 3.2 iii), in ISC, the correct information of returns ratio is always shared between SC members, that is, a manufacturer and a retailer know the correct returns ratio $\alpha^{t}$ of products from a market. Also, from Eq. (6) the expected profit of the whole $\mathrm{SC}$ is a function of order quantity $Q$. .

Therefore, the optimal sales strategy under ISC with information sharing of the correct returns ratio $\alpha^{t}$ is to find the optimal order quantity of product so as to maximize Eq. (6) under the correct returns ratio $\alpha^{t}$. Here, the first-order and second-order differential equations are derived as between $Q$ and the expected profit of the whole SC in Eq. (6) under $\alpha^{t}$ as

$$
\begin{aligned}
& d E\left[\pi_{S}\left(Q \mid \alpha^{t}\right)\right. \\
& \quad=p\left(1-\alpha^{t}\right)+\alpha^{t}\left\{\left(p k-c_{r}\right)+\left(s_{r}-c_{m}\right)(1-k)\right\}+g-c \\
& \quad-\left[p\left(1-\alpha^{t}\right)+\alpha^{t}\left\{\left(p k-c_{r}\right)+\left(s_{r}-c_{m}\right)(1-k)\right\}\right. \\
& \left.\quad+c_{m}+g-s_{r}\right] F(Q), \\
& d^{2} E\left[\pi_{S}\left(Q \mid \alpha^{t}\right)\right] / d Q^{2} \\
& \quad=-\left[p\left(1-\alpha^{t}\right)+\alpha^{t}\left\{\left(p k-c_{r}\right)+\left(s_{r}-c_{m}\right)(1-k)\right\}\right. \\
& \left.\quad+c_{m}+g-s_{r}\right] f(Q) .
\end{aligned}
$$
tions,

Eq. (8) is negative since it is natural that the condi-

$$
\left\{p\left(1-\alpha^{t}\right)+p k \alpha^{t}+s_{r}(1-k)+c_{m}+g\right\}>\left\{c_{r} \alpha^{t}+c_{m}(1-k)+s_{r}\right\}
$$

are generally satisfied. Therefore,

$$
\left\{p\left(1-\alpha^{t}\right)+\alpha^{t}\left\{\left(p k-c_{r}\right)+\left(s_{r}-c_{m}\right)(1-k)\right\}+c_{m}+g-s_{r}\right\}>0
$$

is satisfied. So, the expected profit in Eq. (6) under $\alpha^{t}$ is the concave function in terms of order quantity $Q$. Therefore, by solving Eq. (7) $=0$, the unique optimal order quantity can be determined as

$$
Q^{C}=F^{-1}\left(A^{C} / B^{C}\right),
$$

$$
\begin{aligned}
A^{C}= & p\left(1-\alpha^{t}\right)+\alpha^{t}\left\{\left(p k-c_{r}\right)+\left(s_{r}-c_{m}\right)(1-k)\right\}+g-c, \\
B^{C}= & p\left(1-\alpha^{t}\right)+\alpha^{t}\left\{\left(p k-c_{r}\right)+\left(s_{r}-c_{m}\right)(1-k)\right\} . \\
& +c_{m}+g-s_{r}
\end{aligned}
$$

\section{OPTIMAL SALES STRATEGY UNDER DSC}

This section discusses the optimal sales strategy with and without information sharing of returns ratio between a manufacturer and a retailer under DSC in Buyback policy 1 and Buyback Policy 2, respectively. Under DSC, the manufacturer is the leader of the decision-making and the retailer is the follower of the decision-making. The manufacturer determines the optimal wholesale price so as to maximize the own expected profit. Under the optimal decision by the manufacturer, the retailer determines order quantity so as to maximize the own expected profit.

\subsection{Case with Information Sharing of Returns Ratio under Buy Back Policy $1 \quad(i=1)$}

The first-order and second-order differential equations between order quantity $Q(w)$ and the expected profit of the retailer in Eq. (2) in Buyback Policy 1 under wholesale price $w$ and the correct returns ratio $\alpha^{t}$ are obtained as

$$
\begin{aligned}
d E[ & \left.\pi_{R}^{1}\left(Q(w) \mid w, \alpha^{t}\right)\right] / d Q(w) \\
= & p\left(1-\alpha^{t}\right)+\left(p k-c_{r}\right) \alpha^{t}+b(1-k) \alpha^{t}+g-w \\
- & \left\{p\left(1-\alpha^{t}\right)+\left(p k-c_{r}\right) \alpha^{t}+b(1-k) \alpha^{t}\right. \\
& +g-b\} F\{Q(w)\}, \\
d^{2} E[ & \left.\pi_{R}^{1}\left(Q(w) \mid w, \alpha^{t}\right)\right] / d Q(w)^{2} \\
=- & \left\{p\left(1-\alpha^{t}\right)+\left(p k-c_{r}\right) \alpha^{t}+b(1-k) \alpha^{t}\right. \\
& +g-b\} f\{Q(w)\} .
\end{aligned}
$$

Eq. (13) is negative since it is natural that the conditions $p\left(1-\alpha^{t}\right)+(p-b) k \alpha^{t}>\left(g-c_{r} \alpha^{t}\right)$ are generally satisfied. Therefore, $\left\{p\left(1-\alpha^{t}\right)+\left(p k-c_{r}\right) \alpha^{t}+b(1-k) \alpha^{t}+g\right.$ $-b\}>0$ is satisfied, so the expected profit in Eq. (2) un$\operatorname{der} \alpha^{t}$ is the concave function in terms of order quantity $Q(w)$ under $w$ and $\alpha^{t}$. Solving Eq. (12) $=0$, the tentative optimal order quantity $Q_{D}^{1}\left(w, \alpha^{t}\right)$ under $w$ and $\alpha^{t}$ is determined as

$$
\begin{aligned}
& Q_{D}^{1}\left(w, \alpha^{t}\right)=F^{-1}\left(A_{D}^{1}\left(w, \alpha^{t}\right) / B_{D}^{1}\left(\alpha^{t}\right)\right), \\
& A_{D}^{1}\left(w, \alpha^{t}\right)=p\left(1-\alpha^{t}\right)+\left(p k-c_{r}\right) \alpha^{t}+b(1-k) \alpha^{t}+g-w, \\
& B_{D}^{1}\left(\alpha^{t}\right)=p\left(1-\alpha^{t}\right)+\alpha^{t}\left(p k-c_{r}\right)+b(1-k) \alpha^{t}+g-b .
\end{aligned}
$$

Manufacturer determines the optimal wholesale price $w_{D}^{1}\left(\alpha^{t}\right)$ in Buyback Policy 1 so as to the own expected profit in Eq. (3) under the correct returns ratio 
$\alpha^{t}$ and $Q_{D}^{1}\left(w, \alpha^{t}\right)$, satisfying the following conditions under $\alpha^{t}$ :

$$
\begin{aligned}
& d E\left[\pi_{M}^{1}\left(w, Q_{D}^{1}\left(w, \alpha^{t}\right) \mid \alpha^{t}\right)\right] / d w=0, \\
& d^{2} E\left[\pi_{M}^{1}\left(w, Q_{1}^{D^{*}}\left(w, \alpha^{t}\right) \mid \alpha^{t}\right)\right] / d w^{2}<0 .
\end{aligned}
$$

Substituting $w_{D}^{1}\left(\alpha^{t}\right)$ into Eqs. (14) and (15), the retailer finally determines the optimal order quantity $Q_{D}^{1}$ $\left(w_{D}^{1}\left(\alpha^{t}\right), \alpha^{t}\right)$ in Buyback Policy 1 as

$$
\begin{aligned}
& Q_{D}^{1}\left(w_{D}^{1}\left(\alpha^{t}\right), \alpha^{t}\right)=F^{-1}\left(A_{D}^{1}\left(w_{D}^{1}\left(\alpha^{t}\right), \alpha^{t}\right) / B_{D}^{1}\left(\alpha^{t}\right)\right), \\
& A_{D}^{1}\left(w_{D}^{1}\left(\alpha^{t}\right), \alpha^{t}\right) \\
& \quad=p\left(1-\alpha^{t}\right)+\left(p k-c_{r}\right) \alpha^{t}+b(1-k) \alpha^{t}+g-w_{D}^{1}\left(\alpha^{t}\right) .
\end{aligned}
$$

\subsection{Case with Information Sharing of Returns Ratio under Buy Back Policy $2(i=2)$}

The first-order and second-order differential equations between order quantity $Q(w)$ and the expected profit of the retailer in Eq. (4) in Buyback Policy 2 under wholesale price $w$ and the correct returns ratio $\alpha^{t}$ are obtained as

$$
\begin{aligned}
& d E\left[\pi_{R}^{2}\left(Q(w) \mid w, \alpha^{t}\right)\right] / d Q(w) \\
& =p\left(1-\alpha^{t}\right)+\left(p k-c_{r}\right) \alpha^{t}+b_{r}(1-k) \alpha^{t}+g-w \\
& -\left\{p\left(1-\alpha^{t}\right)+\left(p k-c_{r}\right) \alpha^{t}+b_{r}(1-k) \alpha^{t}\right. \\
& \quad+g-b\} F(Q), \\
& d^{2} E\left[\pi_{R}^{2}\left(Q(w) \mid w, \alpha^{t}\right)\right] / d Q^{2} \\
& =-\left\{p\left(1-\alpha^{t}\right)+\left(p k-c_{r}\right) \alpha^{t}+b_{r}(1-k) \alpha^{t}+g-b\right\} f(Q) .
\end{aligned}
$$

Eq. (22) is negative since it is natural that the conditions $\left\{p\left(1-\alpha^{t}\right)+p k \alpha^{t}+b_{r}(1-k) \alpha^{t}+g\right\}>\left(b+c_{r} \alpha^{t}\right)$ are ge nerally satisfied. Therefore, $\left\{p\left(1-\alpha^{t}\right)+\left(p k-c_{r}\right) \alpha^{t}+b_{r}(1-\right.$ $\left.k) \alpha^{t}+g-b\right\}>0$ is satisfied, so the expected profit in Eq. (4) under $\alpha^{t}$ is the concave function in terms of order quantity $Q(w)$ under $w$ and $\alpha^{t}$. Solving Eq. (21) $=0$, the tentative optimal order quantity $Q_{D}^{2}\left(w, \alpha^{t}\right)$ under $w$ can be determined as

$$
\begin{aligned}
Q_{D}^{2}\left(w, \alpha^{t}\right)= & F^{-1}\left(A_{D}^{2}\left(w, \alpha^{t}\right) / B_{D}^{2}\left(\alpha^{t}\right)\right), \\
A_{D}^{2}\left(w, \alpha^{t}\right)= & p\left(1-\alpha^{t}\right)+\left(p k-c_{r}\right) \alpha^{t} \\
& +b_{r}(1-k) \alpha^{t}+g-w, \\
B_{D}^{2}\left(\alpha^{t}\right)= & p\left(1-\alpha^{t}\right)+\alpha^{t}\left(p k-c_{r}\right)+b_{r}(1-k) \alpha^{t}+g-b .
\end{aligned}
$$

Manufacturer determines the optimal wholesale price $w_{D}^{2}\left(\alpha^{t}\right)$ in Buyback Policy 2 so as to the own expected profit in Eq. (5) under the correct returns ratio $\alpha^{t}$ and $Q_{D}^{2}\left(w, \alpha^{t}\right)$, satisfying the following conditions under $\alpha^{t}$ :

$$
d E\left[\pi_{M}^{2}\left(w, Q_{D}^{2}\left(w, \alpha^{t}\right) \mid \alpha^{t}\right)\right] / d w=0
$$

$d^{2} E\left[\pi_{M}^{2}\left(w, Q_{D}^{2}\left(w, \alpha^{t}\right) \mid \alpha^{t}\right)\right] / d w^{2}<0$.

Substituting $w_{D}^{2}\left(\alpha^{t}\right)$ into Eqs. (23) and (24), the retailer finally determines the optimal order quantity in Buyback Policy 2 as

$$
\begin{aligned}
& Q_{D}^{2}\left(w_{D}^{2}\left(\alpha^{t}\right), \alpha^{t}\right)=F^{-1}\left(A_{D}^{2}\left(w_{D}^{2}\left(\alpha^{t}\right), \alpha^{t}\right) / B_{D}^{2}\left(\alpha^{t}\right)\right), \\
& A_{D}^{2}\left(w_{D}^{2}\left(\alpha^{t}\right), \alpha^{t}\right) \\
& \quad=p(1-\alpha)+\alpha\left\{p k-c_{r}+b_{r}(1-k)\right\}+g-w_{D}^{2}\left(\alpha^{t}\right) .
\end{aligned}
$$

\subsection{Case without Information Sharing of Returns Ratio under Buy Back Policy $1(i=1)$}

Under the case without information sharing of returns ratio between a manufacturer and a retailer under DSC, the manufacturer estimates the returns ratio from Eq. (1). Under the estimated returns ratio of products $\alpha^{e}$. manufacturer estimates the retailer's tentative order quantity so as to maximize the retailer's expected profit by substituting $\alpha^{e}$ into Eqs. (14)-(16). The tentative order quantity $Q_{D}^{1}\left(w, \alpha^{e}\right)$ is determined as

$$
\begin{aligned}
& Q_{D}^{1}\left(w, \alpha^{e}\right)=\left.F^{-1}\left(A_{D}^{1}\left(w, \alpha^{e}\right)\right) / B_{D}^{1}\left(\alpha^{e}\right)\right), \\
& A_{D}^{1}\left(w, \alpha^{e}\right)= p\left(1-\alpha^{e}\right)+\left(p k-c_{r}\right) \alpha^{e} \\
&+b(1-k) \alpha^{e}+g-w, \\
& B_{D}^{1}\left(\alpha^{e}\right)=p\left(1-\alpha^{e}\right)+\alpha^{e}\left(p k-c_{r}\right)+b(1-k) \alpha^{e}+g-b .
\end{aligned}
$$

Manufacturer determines the optimal wholesale price $w_{D}^{1}\left(\alpha^{e}\right)$ in Buyback Policy 1 so as to maximize Eq. (3) under the estimated returns ratio $\alpha^{e}$ and $Q_{D}^{1}\left(w, \alpha^{e}\right)$, satisfying the following conditions under $\alpha^{e}$ :

$$
\begin{aligned}
& d E\left[\pi_{M}^{1}\left(w \mid Q_{D}^{1}\left(w, \alpha^{e}\right), \alpha^{e}\right)\right] / d w=0, \\
& d^{2} E\left[\pi_{M}^{1}\left(w \mid Q_{D}^{1}\left(w, \alpha^{e}\right), \alpha^{e}\right)\right] / d w^{2}<0 .
\end{aligned}
$$

Using $w_{D}^{1}\left(\alpha^{e}\right)$ and Eqs. (16), (30) and (31), the retailer finally determines the optimal order quantity in Buyback Policy 1 as

$$
\begin{aligned}
& \left.Q_{D}^{1}\left(w_{D}^{1}\left(\alpha^{e}\right), \alpha^{t}\right)=F^{-1}\left(A_{D}^{1}\left(w_{D}^{1}\left(\alpha^{e}\right), \alpha^{t}\right)\right) / B_{D}^{1}\left(\alpha^{t}\right)\right), \\
& A_{D}^{1}\left(w_{D}^{1}\left(\alpha^{e}\right), \alpha^{t}\right) \\
& \quad=p\left(1-\alpha^{t}\right)+\left(p k-c_{r}\right) \alpha^{t}+b(1-k) \alpha^{t}+g-w_{D}^{1}\left(\alpha^{e}\right) .
\end{aligned}
$$

\subsection{Case without Information Sharing of Returns Ratio under Buy Back Policy 2 $(i=2)$}

In the similar way as 6.3 , under the estimated returns ratio $\alpha^{e}$, manufacturer estimates the retailer's tentative order quantity $Q_{D}^{2}\left(w, \alpha^{e}\right)$ by substituting $\alpha^{e}$ into Eqs. (23)-(25). The tentative order quantity $Q_{D}^{2}\left(w, \alpha^{e}\right)$ is determined as

$Q_{D}^{2}\left(w, \alpha^{e}\right)=F^{-1}\left(A_{D}^{2}\left(w, \alpha^{e}\right) / B_{D}^{2}\left(\alpha^{e}\right)\right)$, 


$$
\begin{aligned}
A_{D}^{2}\left(w, \alpha^{e}\right)= & p\left(1-\alpha^{e}\right)+\left(p k-c_{r}\right) \alpha^{e} \\
& +b_{r}(1-k) \alpha^{e}+g-w \\
B_{D}^{2}\left(\alpha^{e}\right)= & p\left(1-\alpha^{e}\right)+\alpha^{e}\left(p k-c_{r}\right)+b_{r}(1-k) \alpha^{e}+g-b .
\end{aligned}
$$

Manufacturer determines the optimal wholesale price $w_{D}^{2}\left(\alpha^{e}\right)$ in Buyback Policy 2 so as to maximize Eq. (6) under the estimated returns ratio $\alpha^{e}$ and $Q_{D}^{2}\left(w, \alpha^{e}\right)$, satisfying the following conditions under $\alpha^{e}$ :

$d E\left[\pi_{M}^{2}\left(w \mid Q_{D}^{2}\left(w, \alpha^{e}\right), \alpha^{e}\right)\right] / d w=0$,

$d^{2} E\left[\pi_{M}^{2}\left(w \mid Q_{D}^{2}\left(w, \alpha^{e}\right), \alpha^{e}\right)\right] / d w^{2}<0$.

Using $w_{D}^{2}\left(\alpha^{e}\right)$ and Eqs. (25), (37) and (38), the retailer finally determines the optimal order quantity in Buyback Policy 2 as

$$
\begin{aligned}
& Q_{D}^{2}\left(w_{D}^{2}\left(\alpha^{e}\right), \alpha^{t}\right)=F^{-1}\left(A_{D}^{2}\left(w_{D}^{2}\left(\alpha^{e}\right), \alpha^{t}\right) / B_{D}^{2}\left(\alpha^{t}\right)\right), \\
& A_{D}^{2}\left(w_{D}^{2}\left(\alpha^{e}\right), \alpha^{t}\right) \\
& =p\left(1-\alpha^{t}\right)+\left(p k-c_{r}\right) \alpha^{t}+b_{r}(1-k) \alpha^{t}+g-w_{D}^{2}\left(\alpha^{e}\right) .
\end{aligned}
$$

\section{NUMERICAL ANALYSIS}

This section illustrates results of the optimal sales strategies under DSC and ISC in buyback policy 1 and buyback policy 2 , considering the difference of quality of products bought back from a retailer and the state of information sharing of customer returns ratio by providing numerical examples. Concretely, the analysis numerically investigates how information sharing of the customer returns ratio affects the optimal sales strategies and the expected profits under ISC and DSC in each buyback policy. Also, the results under ISC are compared with those under DSC. Here, the profitability of information sharing of the customer returns ratio is evaluated by comparing the expected profits under each SC without information sharing with those with it. Moreover, SC coordination to guarantee the profit improvement for each SC member under ISC is discussed. Concretely, the wholesale price and the buyback price of the unsold products and the un-reusable products between the retailer and the manufacturer are coordinated as Nash bargaining solutions. The profitability of profit sharing on SC members under ISC is verified by comparing with their expected profits under DSC.

Data sources of the numerical examples are provided as follows: $p=80, s=10, s_{r}=5, c=20, k=0.8, b=35$, $b_{r}=15, g=5, c_{r}=1, c_{m}=1$. The product demand $x$ follows the normal distribution with the mean $\mu=1000$ and variance $\sigma^{2}=300$. The correct returns ratio is $\alpha^{t}=$ 0.2 and the estimated returns ratio $\alpha^{e}$ by a manufacturer is varied from 0.02 to 0.4 at a step size 0.02 . All data sources of the numerical examples in this paper above are provided so as to satisfy the conditions of $\alpha^{j}(j=t$, e), $k$ and model assumptions in 3.2 (iv) as

$$
\begin{aligned}
& p>w>c \geq g \geq s_{r}>c_{r} \geq c_{m}, w>b>b_{r}, \\
& 0 \leq \alpha^{j} \leq 1(j=t, e), 0 \leq k \leq 1,
\end{aligned}
$$

guaranteeing profitable operations of a retailer, a manufacturer and the whole SC under e-commerce. Also, all data sources of the numerical examples satisfying the above conditions are modifiable if necessary to conduct sensitive analysis.

A computer programming was developed by using Visual Studio C\# in Visual Studio Express 2013 for Windows Desktop in order to conduct numerical experiments and obtain the results for the optimal sales strategies under DSC and ISC under e-commerce by the numerical calculation and the numerical search. In the development of the computer programming and implementation of the numerical experiment, the following computer : the Dell computer, Vostro 260s model, CPU: Intel(R) Core(TR) i5-2400, 3.10 GHz: Memory: 4 GB, OS: Windows 7 Professional 32bit was used in this paper.

\subsection{Comparison of Optimal Sales Strategy and Expected Profits in Buyback Policy 1 and Buyback Policy 2}

Here, the optimal sales strategy and the expected profits in buyback policy 1 is compared with those in buyback policy 2 under the correct returns ratio. Table 1 shows the optimal decisions for the wholesale price, the order quantity and the expected profits under DSC in buyback policies 1 and 2 under the correct returns ratio $\alpha^{t}=0.2$. Also, Table 1 shows the optimal decision for the order quantity and the expected profits under ISC under the correct returns ratio $\alpha^{t}=0.2$. From Table 1, the following results can be seen: the optimal order quantity under ISC in buyback policies 1 and 2 is larger than that under DSC. This is because production cost and salvage value are generally lower than wholesale price and buyback price. The optimal decisions under DSC in buyback policy 2 bring the more expected prof-

Table 1. Optimal decisions and the expected profits under DSC and ISC under correct returns ratio $\alpha^{t}=0.2$

\begin{tabular}{cccccc}
\hline Correct returns ratio $\alpha^{t}=0.2$ & Wholesale price & Order quantity & Retailer & Manufacturer & Whole SC \\
\hline DSC in buyback policy 1 without considering quality & 71 & 799 & 2,404 & 38,469 & 40,873 \\
\hline DSC in buyback policy 2 with considering quality & 70 & 807 & 2,601 & 38,596 & 41,197 \\
\hline ISC & - & 1,246 & 73,772 & $-23,650$ & 50,122 \\
\hline
\end{tabular}


its to a manufacturer, a retailer and the whole SC than those under DSC in buyback policy 1. Therefore, the numerical analysis focus on comparison of results under DSC in buyback policy 2 with those under ISC.

\subsection{Value of Information Sharing of Returns Ratio between A Manufacturer and A Retailer under DSC}

Here, the value of information sharing of returns ratio between a manufacturer and a retailer under DSC in buyback policy 2 is investigated. Figure 1 shows the effect of information sharing of customer returns ratio on the optimal order quantity and the optimal wholesale price. The $\mathrm{x}$-axis shows the ratio $\alpha^{e} / \alpha^{t}$ of the estimated customer returns to the correct one. The situation where $\alpha^{e} / \alpha^{t}>1$ indicates that a manufacturer estimates the customer returns ratio of products as a higher value $\alpha^{e}$ than $\alpha^{t}$.

From Figure 1, the following results can be seen: the higher a manufacturer estimates the customer returns ratio of products, the lower the optimal wholesale price the manufacturer made, meanwhile the more product.

Figure 2 shows the value of information sharing of customer returns ratio between a retailer and a manufacturer. In this paper, the values of information sharing of returns ratio between a manufacturer and a retailer, $V_{M}^{2}$ and $V_{R}^{2}$ are calculated as

$$
\begin{gathered}
V_{M}^{2}=E\left[\pi_{M}^{2}\left(\alpha^{t} \mid w_{D}^{2}\left(\alpha^{t}\right), Q_{D}^{2}\left(\alpha^{t}\right)\right)\right] \\
-E\left[\pi_{M}^{2}\left(\alpha^{t} \mid w_{D}^{2}\left(\alpha^{e}\right), Q_{D}^{2}\left(\alpha^{e}\right)\right)\right], \\
V_{R}^{2}=E\left[\pi_{R}^{2}\left(\alpha^{t} \mid w_{D}^{2}\left(\alpha^{t}\right), Q_{D}^{2}\left(\alpha^{t}\right)\right)\right] \\
-E\left[\pi_{R}^{2}\left(\alpha^{t} \mid w_{D}^{2}\left(\alpha^{e}\right), Q_{D}^{2}\left(\alpha^{e}\right)\right)\right] .
\end{gathered}
$$

From Figure 2, the following results can be seen: the expected profit of a manufacturer is not highly affected by the situation with or without information sharing of the customer returns ratio with a retailer. This is because a manufacturer is the leader of decision-making under DSC and can decide the optimal wholesale price so as to maximize the own expected profit, considering the situation with or without information sharing of the customer returns ratio with a retailer. On the other hand, it is verified that the smaller the value of $\alpha^{e} / \alpha^{t}$ is, the higher the optimal wholesale price is and the smaller the optimal order quantity is. These results bring profit loss to the retailer. Therefore, it is necessary for retailer to share the customer returns ratio where $\alpha^{e} / \alpha^{t}<1$, which indicates that a manufacturer estimates the customer returns ratio of products as a higher value $\alpha^{e}$ than $\alpha^{t}$, meanwhile it is not necessary where $\alpha^{e} / \alpha^{t}<1$. Figures 1 and 2 verified that it is necessary for a retailer to share the customer returns ratio with a manufacturer so as to avoid unprofitable situation for the retailer due to the estimated returns ratio by a manufacturer.

Moreover, the expected profits with the correct customer returns ratio under DSC are compared with those under ISC. Table 2 shows the expected profits under DSC and ISC in buyback policy 2 under the correct customer returns ratio $\alpha^{t}=0.2$. From Table 2 , the expected profits of a retailer and the whole SC under ISC are higher than those under DSC, meanwhile the expected profit of a manufacturer is negative under ISC, since the wholesale price is canceled out between a manufacturer and a retailer under ISC. Under the situation, it is impossible for a manufacturer to shift the optimal decision under ISC from that under DSC. So, it is necessary for a manufacturer who is the leader of the decision-making under DSC to make SCC between a manufacturer and a retailer under ISC in order to encourage the shift the optimal decision under ISC from that under DSC.

\subsection{Supply Chain Coordination in SC under E-Commerce}

This paper adopts the profit sharing to coordinate the unit wholesale price $w$ and the buyback prices, $b$ and $b_{r}$, between the manufacturer and the retailer under ISC in buyback policy 2. Concretely, in buyback policy 2 , the unit wholesale price $w$ and buyback prices, $b$ and $b_{r}$, between a manufacturer and a retailer are coordinated as the Nash bargaining solutions (Nagarajan and Sosic, 2008; Du et al., 2011). The coordinated prices $\left(w^{N}\left(\alpha^{j}\right), b^{N}\left(\alpha^{j}\right), b_{r}^{N}\left(\alpha^{j}\right)\right)$ are determined so as to satisfy

\begin{tabular}{|c|c|c|c|c|c|c|c|}
\hline \multirow{2}{*}{$\begin{array}{c}\text { Correct returns } \\
\quad \text { ratio } \\
\alpha^{t}=0.2\end{array}$} & \multirow{2}{*}{$\begin{array}{l}\text { Wholesale } \\
\text { price }\end{array}$} & \multirow{2}{*}{$\begin{array}{l}\text { Order } \\
\text { quantity }\end{array}$} & \multirow{2}{*}{$\begin{array}{l}\text { Buyback price } b \\
\text { of unsold products }\end{array}$} & \multirow{2}{*}{$\begin{array}{l}\text { Buyback price } b_{r} \\
\text { of un-resold } \\
\text { returned products }\end{array}$} & \multicolumn{3}{|c|}{ Expected profits } \\
\hline & & & & & Manufacturer & Retailer & Whole SC \\
\hline DSC & 70 & 807 & 35 & 15 & 38,596 & 2,601 & 41,197 \\
\hline ISC & - & 1,246 & - & - & $-23,650$ & 73,772 & 50,122 \\
\hline ISC with SCC & 66 & 1,246 & 52 & 25 & 43,059 & 7,063 & 50,122 \\
\hline
\end{tabular}
the following equations under returns ratio $\alpha^{j}(j=t, e)$ :

$$
\begin{aligned}
& \max _{w^{N}\left(\alpha^{j}\right), b^{N}\left(\alpha^{j}\right), b_{r}^{N}\left(\alpha^{j}\right)} \pi\left(w^{N}\left(\alpha^{j}\right), b^{N}\left(\alpha^{j}\right), b_{r}^{N}\left(\alpha^{j}\right) \mid \alpha^{j}\right)(j=t, e) \\
& =\left\{E\left[\pi_{R}^{2}\left(w^{N}\left(\alpha^{j}\right), b^{N}\left(\alpha^{j}\right), b_{r}^{N}\left(\alpha^{j}\right) \mid Q_{C}, \alpha^{j}\right)\right]\right. \\
& \left.-E\left[\pi_{R}^{2}\left(w_{D}^{2}\left(\alpha^{j}\right), b, b_{r}, Q_{D}^{2}\left(w_{D}^{2}\left(\alpha^{j}\right), \alpha^{j}\right) \mid \alpha^{j}\right)\right]\right\} \\
& \times\left\{E\left[\pi_{M}^{2}\left(w^{N}\left(\alpha^{j}\right), b^{N}\left(\alpha^{j}\right), b_{r}^{N}\left(\alpha^{j}\right) \mid Q_{C}, \alpha^{j}\right)\right]\right.
\end{aligned}
$$

Table 2. the expected profits under DSC and ISC in buyback policy 2 under the correct customer returns ratio $\alpha^{t}=0.2$ 


$$
\begin{aligned}
- & \left.E\left[\pi_{M}^{2}\left(w_{D}^{2}\left(\alpha^{j}\right), b, b_{r}, Q_{D}^{2}\left(w_{D}^{2}\left(\alpha^{j}\right), \alpha^{j}\right) \mid \alpha^{j}\right)\right]\right\}, \\
\{E & {\left[\pi_{R}^{2}\left(w^{N}\left(\alpha^{j}\right), b^{N}\left(\alpha^{j}\right), b_{r}^{N}\left(\alpha^{j}\right) \mid Q_{C}, \alpha^{j}\right)\right] } \\
& \left.-E\left[\pi_{R}^{2}\left(w_{D}^{2}\left(\alpha^{j}\right) b, b_{r}, Q_{D}^{2}\left(w_{D}^{2}\left(\alpha^{j}\right), \alpha^{j}\right) \mid \alpha^{j}\right)\right]\right\}>0, \\
\{E & {\left[\pi_{M}^{2}\left(w^{N}\left(\alpha^{j}\right), b^{N}\left(\alpha^{j}\right), b_{r}^{N}\left(\alpha^{j}\right) \mid Q_{C}, \alpha^{j}\right)\right] } \\
& \left.-E\left[\pi_{M}^{2}\left(w_{D}^{2}\left(\alpha^{j}\right) b, b_{r}, Q_{D}^{2}\left(w_{D}^{2}\left(\alpha^{j}\right), \alpha^{j}\right) \mid \alpha^{j}\right)\right]\right\}>0 .
\end{aligned}
$$

Here, Eqs. (47) and (48) are constraint conditions to guarantee that the expected profits of both members under ISC with SCC are always higher than those under DSC.

In Table 2, the expected profits with the correct customer returns ratio under ISC with SCC are compared with those under DSC. It is verified that SCC re-

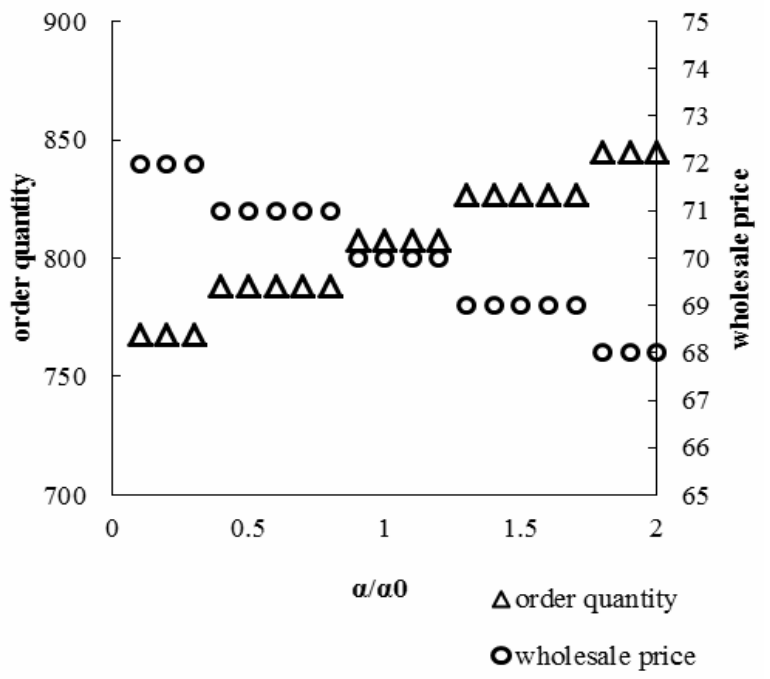

Figure 1. Effect of information sharing of customer returns ratio on optimal order quantity and optimal wholesale price.

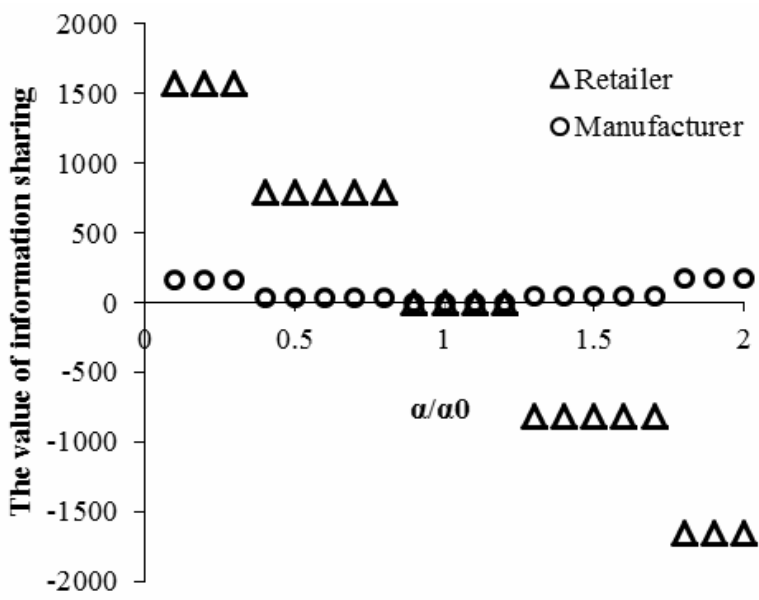

Figure 2. Value of information sharing of customer returns ratio between a retailer and a manufacturer. garding the unit wholesale price and buyback prices between a manufacturer and a retailer under ISC bring the more expected profits to a manufacturer and a retailer under ISC than those under DSC. Thus, SCC under ISC enables to guarantee the profit improvement for a manufacturer and a retailer under ISC and encourage the shift the optimal decision under ISC from that under DSC.

\section{CONCLUSIONS}

This paper discussed the effect of information sharing regarding customer returns on the optimal sales strategy under e-commerce for the integrated supply chain (ISC) and the decentralized supply chain (DSC). SC members consisted of a retailer and a manufacturer. In ISC, the optimal decision for product order quantity was made to maximize the expected profit of the whole SC. In DSC, the optimal decisions for product order quantity and the product wholesale price were made to maximize the expected profit of each SC member. This paper combined the following topics: (i) resale of product returns and (ii) manufacturer's buyback policy without/with difference of the unit buyback price and (iii) supply chain coordination (SCC) under ISC. Also, the effect of information sharing regarding customer returns on the optimal decisions of ISC and DSC was discussed. As the manufacturer's buyback policy, buyback policy 1 without difference of the unit buyback price between the unsold products and the customer returns and buyback policy 2 with it were considered. The unit wholesale price and buyback prices were adjusted between SC members under the optimal sales strategy of ISC as Nash bargain solutions. The research outcomes of this paper were summarize as follows:

- The optimal order quantity under ISC with each buyback policy is larger than that under DSC. The optimal decision under DSC with buyback policy 2 brought the more expected profits to SC members and the whole SC than those under DSC in buyback policy 1.

- The expected profit of a manufacturer, the leader of decision-making under DSC, was not highly affected by information sharing of the customer returns ratio of a retailer.

- It is necessary for a retailer to share the customer returns ratio with a manufacturer so as to avoid an unprofitable situation due to the estimated returns ratio by a manufacturer.

- SCC by adjusting the unit wholesale price and buyback prices under ISC could encourage the shift the optimal sales strategy under ISC from that under DSC

Thus, it assured that not only finding of this paper can connect strongly to E-business and those profitable management, but also they can provide not only informative motivation, but also one optimal solution to con- 
struct and operate profitably a supply chain under e-commerce for researchers and policymakers who engage in the optimal operations regarding supply chains.

As future researches, it will be necessary to incorporate the following extendable topics into the SC model under e-commerce discussed in this paper:

- Impact of the unit sales price, refund of customer returns and delivery lead time of products on product demands;

- Impact of either the unit sales price or delivery lead time of products on a product order quantity of a retailer;

- Situation where the multiple types of products are handled in a supply chain;

- Situation where the probability distribution of product demand is unknown

- Impact of supply disruptions on the optimal sales strategy in a SC under e-commerce;

- Proposal of an alternative approach of profit sharing as supply chain coordination to promote a retailer-manufacturer partnership by combing cooperative game models with the proposed profit sharing approach;

- Applications of research results of this paper with case study of firms with similar distribution of a supply chain; and

- Applications of research results of this paper to service sciences and management.

\section{ACKNOWLEDGMENTS}

This research has been supported by the Grant-inAid for Scientific Research C No. 25350451 from the Japan Society for the Promotion of Science.

\section{REFERENCES}

Aust, G. and Buscher, U. (2012), Vertical cooperative advertising and pricing decisions in a manufacturerretailer supply chain: a game-theoretic approach, European Journal of Operational Research, 223(2), 473-482.

Berr, F. (2011), Stackelberg equilibria in managerial delegation games, European Journal of Operational Research, 212(2), 251-262.

Cai, G. G., Zhang, Z. G., and Zhang, M. (2009), Game theoretical perspectives on dual-channel supply chain competition with price discounts and pricing schemes, International Journal of Production Economics, 117(1), 80-96.
Cachon, G. P. and Netessine, S. (2004), Game theory in supply chain analysis. In: Simchi-Levi, D., Wu, S. D., and Shen, Z. J. (eds.), Handbook of Quantitative Supply Chain Analysis: Modeling in the e-Business Era, Kluwer, Boston, MA, 13-65.

Chauhan, S. S. and Proth, J. M. (2005), Analysis of a supply chain partnership with revenue sharing, International Journal of Production Economics, 97, 44-51.

Chen, J. and Bell, P. C. (2009), The impact of customer returns on pricing and order decisions, European Journal of Operational Research, 195, 280-295.

Chen, J. (2011), The impact of Sharing Customer returns Information in a Supply Chain with and without a Buyback Policy, European Journal of Operational Research, 213, 478-488.

Choi, T. M., Li, D., and Yan H. (2004), Optimal returns policy for supply chain with e-marketplace, International Journal of Production Economics, 88, 205227.

Du, J., Liang, L., Chen, Y., Cook, W. D., and Zhu, J. (2011), A bargaining game model for measuring performance of two-stage network structures, European Journal of Operational Research, 210(2), 390397.

Kusukawa, E., Niimi, Y., and Arizono, I. (2009), Optimal Selling Policy for a Supply Chain System under an E-Commerce Environment with Return Handling. In: Takeyasu, K. and Tsuji, H. (eds.), Management information system and its applications, Osaka Municipal Universities Press, Osaka, Japan, 180-201.

$\mathrm{Hu}$, Y., Guan, Y., and Liu, T. (2011), Lead-time hedging and coordination between manufacturing and sales departments using Nash and Stackelberg games, European Journal of Operational Research, 210(2), 231-240.

Lee, C. H. (2001), Coordinated stocking, clearance sales, and return policies for a supply chain, European $\mathrm{JO}_{\mathrm{O}}$ urnal of Operational Research, 131, 491-513.

Lee, C. H. (2007), Coordination on stocking and progressive pricing policies for a supply chain, International Journal of Production Economics, 106, 307319.

Nagarajan, M. and Sosic, G. (2008), Game-theoretic analysis of cooperation among supply chain agents: review and extensions, European Journal of Operational Research, 187(3), 719-745.

Vlachos, D. and Dekker, R. (2003), Return Handling Options and Order Quantities for Single Period Products, European Journal of Operational Research, 151, 38-52. 